\title{
ON BASIN OF ZERO-SOLUTIONS TO \\ A SEMILINEAR PARABOLIC EQUATION \\ WITH ORNSTEIN-UHLENBECK OPERATOR
}

YASUHIRO FUJITA

Received 27 April 2005; Accepted 10 July 2005

We consider the basin of the zero-solution to a semilinear parabolic equation on $\mathbb{R}^{N}$ with the Ornstein-Uhlenbeck operator. Our aim is to show that the Ornstein-Uhlenbeck operator contributes to enlargement of the basin by using the logarithmic Sobolev inequality.

Copyright (c) 2006 Yasuhiro Fujita. This is an open access article distributed under the Creative Commons Attribution License, which permits unrestricted use, distribution, and reproduction in any medium, provided the original work is properly cited.

\section{Introduction}

Let $\alpha, \beta>0$ be given constants. We consider the following semilinear parabolic problem:

$$
\begin{gathered}
u_{t}=\frac{1}{2} \Delta u-\alpha x \cdot D u+\beta u \log u \quad \text { in }(0, \infty) \times \mathbb{R}^{N}, \\
u(0, \cdot)=\varphi \quad \text { in } \mathbb{R}^{N},
\end{gathered}
$$

where the initial data $\varphi$ satisfies

$$
\varphi>0 \quad \text { in } \mathbb{R}^{N}, \quad \psi:=\log \varphi \in \operatorname{Lip}\left(\mathbb{R}^{N}\right) .
$$

When $\alpha=0$, problem (1.1) was considered by Samarskii et al. in [8, pages 93-99]. When $\alpha>0$, the operator $L$ defined by

$$
L=\frac{1}{2} \Delta-\alpha x \cdot D
$$

is called the Ornstein-Uhlenbeck operator and has been studied by many authors ([1$4,6]$ ). In linear parabolic equations, the Ornstein-Uhlenbeck operator contributes good properties to their solutions such as ergodicity and hypercontractivity. However, to semilinear parabolic equations, a contribution of the Ornstein-Uhlenbeck operator is hardly known. 
Our motivation to study problem (1.1) is that it provides an example of semilinear parabolic equations to which the Ornstein-Uhlenbeck operator contributes. Indeed, in (1.1), the Ornstein-Uhlenbeck operator $L$ contributes to enlargement of the basin of the zero-solution.

Our aim of this paper is to clarify this contribution by using the relation between the parameters $\alpha, \beta$. Our result states that if $\alpha$ is sufficiently larger than $\beta / 2$ then the basin of the zero-solutions is large enough; on the other hand, if $\alpha$ is sufficiently smaller than $\beta / 2$ then it is small enough. Note that as $\alpha$ increases the attractive power to the origin is stronger in the Ornstein-Uhlenbeck operator. Hence, the results above show that enlargement of the basin arises from a contribution of the Ornstein-Uhlenbeck operator.

The contents of the paper are organized as follows: in Section 2, we state existence and uniqueness of a classical solution to (1.1). In Section 3, we derive $L^{q}$-estimates of the classical solution to (1.1). These estimates are based on the logarithmic Sobolev inequality and the Jensen inequality. In Section 4, we state our main results and prove them.

\section{A classical solution to (1.1)}

In this section, we will show existence and uniqueness of a classical solution to (1.1). In order to show existence and uniqueness of a classical solution to (1.1), we consider first the following semilinear parabolic problem:

$$
\begin{array}{cl}
\eta_{t}=\frac{1}{2} \Delta \eta-\alpha x \cdot D \eta+\frac{1}{2} e^{\beta t}|D \eta|^{2} & \text { in }(0, \infty) \times \mathbb{R}^{N}, \\
\eta(0, \cdot)=\psi(\cdot):=\log \varphi(\cdot) & \text { in } \mathbb{R}^{N} .
\end{array}
$$

Note that $\eta$ is a classical solution to (2.1) if and only if the function $u$ defined by

$$
u=\exp \left(e^{\beta t} \eta\right)
$$

is a classical solution to (1.1). For this reason, we consider (2.1). When the time-dependent Hamiltonian $e^{\beta t}|D \eta|^{2} / 2$ of (2.1) is replaced by the time-independent Hamiltonian $H(D \eta)$ for some $H \in C^{1}\left(\mathbb{R}^{N}\right)$, existence and uniqueness of a classical solution to (2.1) was shown in [6]. Our proof for (2.1) is almost same as that of [6]. So, we omit it. Let

$$
Q=(0, \infty) \times \mathbb{R}^{N}
$$

Theorem 2.1 ([6]). Assume (1.2). Then, (2.1) admits at least one classical solution $\eta$ such that $\eta \in C(\bar{Q}) \cap C^{1,2}(Q)$ with the property

$$
\left\|D_{x} \eta\right\|_{\infty, Q}<\infty
$$

Now, we state existence and uniqueness of a classical solution to (1.1).

Theorem 2.2. Assume (1.2). Then (2.1) admits the unique classical solution $u \in C(\bar{Q}) \cap$ $C^{1,2}(Q)$ satisfying the following: $u(\cdot)>0$ in $Q$, and for each $T>0$ there exists a constant $C_{T}>0$ satisfying

$$
|D \log u(t, x)| \leq C_{T}, \quad(t, x) \in(0, T] \times \mathbb{R}^{N}
$$


Proof. Existence of $u$ satisfying the theorem follows from Theorem 2.1. Let $u_{1}$ and $u_{2}$ be such solutions. Let $\eta_{j}=e^{-\beta t} \log u_{j}$. Then $\eta_{j}$ satisfies

$$
\begin{gathered}
\left(\eta_{j}\right)_{t}=\frac{1}{2} \Delta \eta_{j}-\alpha x \cdot D \eta_{j}+\frac{1}{2} e^{\beta t}\left|D \eta_{j}\right|^{2} \quad \text { in }(0, \infty) \times \mathbb{R}^{N}, \\
\eta_{j}(0, \cdot)=\psi(\cdot) \text { in } \mathbb{R}^{N} .
\end{gathered}
$$

Hence, we obtain in $(0, \infty) \times \mathbb{R}^{N}$,

$$
\left(\eta_{1}-\eta_{2}\right)_{t}=\frac{1}{2} \Delta\left(\eta_{1}-\eta_{2}\right)+\left[-\alpha x+\frac{1}{2} e^{\beta t}\left(D \eta_{1}+D \eta_{2}\right)\right] \cdot D\left(\eta_{1}-\eta_{2}\right) .
$$

Note that, for each $T>0$, there exists a constant $K_{T}>0$ such that

$$
\begin{gathered}
\left|-\alpha x+\frac{1}{2} e^{\beta t}\left(D \eta_{1}+D \eta_{2}\right)(t, x)\right| \leq K_{T}(1+|x|), \quad(t, x) \in(0, T] \times \mathbb{R}^{N}, \\
\left|\left(\eta_{1}-\eta_{2}\right)(t, x)\right| \leq K_{T}(1+|x|), \quad(t, x) \in[0, T] \times \mathbb{R}^{N} .
\end{gathered}
$$

Hence, by the comparison theorem for parabolic equations (cf. [5, Theorem 9, page 43]), we deduce that $\eta_{1} \equiv \eta_{2}$ on $[0, T] \times \mathbb{R}^{N}$. Since $T>0$ is arbitrarily, we conclude the theorem. The proof is complete.

\section{3. $L^{q}$-estimates of the solution to (1.1)}

In this section, we will give $L^{q}$-estimates of the unique classical solution to (1.1). Let $v$ be the Borel probability measure on $\mathbb{R}^{N}$ defined by

$$
d \nu(y)=(\alpha / \pi)^{N / 2} e^{-\alpha|y|^{2}} d y .
$$

This measure is called the invariant probability measure for the Ornstein-Uhlenbeck operator $L$ of (1.3), because we have

$$
\int_{\mathbb{R}^{N}} L \chi d v=0, \quad \chi \in C_{b}^{2}\left(\mathbb{R}^{N}\right)
$$

(see $[2,3])$. We give the logarithmic Sobolev inequality without proof (cf. [7]).

Lemma 3.1 [7]. For any $q>1$ and $0<\chi \in C_{b}^{2}\left(\mathbb{R}^{N}\right)$, we have

$$
\int_{\mathbb{R}^{N}} \chi^{q} \log \chi^{q} d \nu \leq-\frac{q^{2}}{2 \alpha(q-1)} \int_{\mathbb{R}^{N}} \chi^{q-1} L \chi d \nu+\|\chi\|_{L^{q}(v)}^{q} \log \|\chi\|_{L^{q}(v)}^{q} .
$$

Next, we have the following lemma.

Lemma 3.2. . For any $q>1$ and $0<\chi \in C_{b}^{2}\left(\mathbb{R}^{N}\right)$,

$$
\int_{\mathbb{R}^{N}} \chi^{q-1} L \chi d v \leq 0
$$


4 On the basin of zero-solutions

Proof. Let $\chi_{n}(x)=\chi(x)+(1 / n)$ for $n \in \mathbb{N}$. Since $\chi_{n}^{q} \in C_{b}^{2}\left(\mathbb{R}^{N}\right)$, it follows from (3.2) that

$$
\int_{\mathbb{R}^{N}} L\left[\chi_{n}^{q}\right] d \nu=0
$$

Since

$$
L\left[\chi_{n}^{q}\right]=q \chi_{n}^{q-1} L \chi+\frac{1}{2} q(q-1) \chi_{n}^{q-2}|D \chi|^{2}
$$

we obtain

$$
\int_{\mathbb{R}^{N}} \chi_{n}^{q-1} L \chi d \nu \leq 0
$$

We conclude (3.4) from the Lebesgue's dominated convergence theorem.

The following proposition follows easily from Theorem 2.2.

Lemma 3.3. Assume (1.2). Let u be the unique classical solution to (1.1) obtained in Theorem 2.2. Then, for any $T>0$, there exists a constant $C_{T}>0$ such that

$$
\begin{gathered}
e^{-C_{T}(1+|x|)} \leq u(t, x) \leq e^{C_{T}(1+|x|)}, \quad(t, x) \in[0, T] \times \mathbb{R}^{N} \\
|D u(t, x)| \leq e^{C_{T}(1+|x|)}, \quad(t, x) \in(0, T] \times \mathbb{R}^{N}
\end{gathered}
$$

Now, we state the main results of this section.

Theorem 3.4. . Assume that (1.2) holds and $2 \alpha>\beta$. Let $u$ be the unique classical solution to (1.1) obtained in Theorem 2.2. Then, for any $q \geq 2 \alpha /(2 \alpha-\beta)$,

$$
\|u(t, \cdot)\|_{L^{q}(v)} \leq \exp \left\{e^{\beta t} \log \|\varphi\|_{L^{q}(v)}\right\}, \quad t \geq 0 .
$$

Proof. Let $\rho \in C^{\infty}\left(\mathbb{R}^{N}\right)$ be a function such that $0 \leq \rho(\cdot) \leq 1$ and

$$
\rho(x)= \begin{cases}1, & |x| \leq 1 \\ 0, & |x| \geq 2 .\end{cases}
$$

We set

$$
\rho_{n}(x)=\rho\left(\frac{x}{n}\right)
$$

Now, we define the function $u_{n}$ by

$$
u_{n}(t, x)=u(t, x) \rho_{n}(x)
$$

Note that

$$
\begin{gathered}
\left(u_{n}^{q}\right)_{t}=q u_{n}^{q-1} \rho_{n}(L u+\beta u \log u), \\
\rho_{n} L u=L u_{n}-u L \rho_{n}-D u \cdot D \rho_{n}, \\
u_{n}^{q} \log u=u_{n}^{q} \log u_{n}-u_{n}^{q} \log \rho_{n} .
\end{gathered}
$$


Here and henceforth, we interpret that $0 \log 0=0$. Using these equalities, we get for $q \geq$ $2 \alpha /(2 \alpha-\beta)$,

$$
\begin{aligned}
\frac{d}{d t}\left\|u_{n}(t, \cdot)\right\|_{L^{q}(v)}^{q} & \\
= & q\left[\int_{\mathbb{R}^{N}} u_{n}(t, \cdot)^{q-1} L u_{n}(t, \cdot) d \nu+\beta \int_{\mathbb{R}^{N}} u_{n}(t, \cdot)^{q} \log u_{n}(t, \cdot) d \nu\right] \\
& -q \int_{\mathbb{R}^{N}}\left[u_{n}(t, \cdot)^{q-1}\left(u(t, \cdot) L \rho_{n}+D u(t, \cdot) \cdot D \rho_{n}\right)+\beta u_{n}(t, \cdot)^{q} \log \rho_{n}\right] d \nu \\
:= & I(t)-J(t) .
\end{aligned}
$$

Since $1 \geq q \beta / 2 \alpha(q-1)$, we have by Lemmas 3.1 and 3.2

$$
\begin{aligned}
I(t) & \leq q\left[1-\frac{q \beta}{2 \alpha(q-1)}\right] \int_{\mathbb{R}^{N}} L u_{n}(t, \cdot) u_{n}(t, \cdot)^{q-1} d v+\beta\left\|u_{n}(t, \cdot)\right\|_{L^{q}(v)}^{q} \log \left\|u_{n}(t, \cdot)\right\|_{L^{q}(v)}^{q} \\
& \leq \beta\left\|u_{n}(t, \cdot)\right\|_{L^{q}(v)}^{q} \log \left\|u_{n}(t, \cdot)\right\|_{L^{q}(v)}^{q}, \quad t>0 .
\end{aligned}
$$

Next, let us fix $T>0$ arbitrarily. By Lemma 3.3, it is easy to see that

$$
\theta_{n}(T):=\sup \{|J(t)| \mid t \in[0, T]\} \longrightarrow 0 \quad \text { as } n \longrightarrow \infty .
$$

Then the function $f_{n}(t)$ defined by

$$
f_{n}(t)=\left\|u_{n}(t, \cdot)\right\|_{L^{q}(v)}^{q}
$$

satisfies

$$
\frac{d}{d t} f_{n}(t) \leq \beta f_{n}(t) \log f_{n}(t)+\theta_{n}(T), \quad 0<t<T
$$

Note that since $\operatorname{supp} \rho_{n} \supset\{x|| x \mid \leq 1\}$ for all $n \geq 1$, we have

$$
f_{n}(t) \geq \int_{\{|x| \leq 1\}} u(t, x)^{q} d \nu \geq \int_{\{|x| \leq 1\}} e^{-q C_{T}(1+|x|)} d \nu=: \gamma_{T}>0, \quad 0 \leq t \leq T,
$$

in view of Lemma 3.3. Then, by (3.18), we obtain

$$
\frac{d}{d t} \log f_{n}(t) \leq \beta \log f_{n}(t)+\frac{\theta_{n}(T)}{\gamma_{T}}, \quad 0<t<T .
$$

From this inequality, we have

$$
e^{-\beta t} \log \left\|u_{n}(t, \cdot)\right\|_{L^{q}(v)}^{q} \leq \log \left\|\chi_{n} \varphi\right\|_{L^{q}(v)}^{q}+\frac{\theta_{n}(T)}{\beta \gamma_{T}}\left(1-e^{-\beta t}\right), \quad 0 \leq t \leq T .
$$


Letting $n \rightarrow \infty$ and using the Lebesgue's dominated convergence theorem, we conclude that

$$
e^{-\beta t} \log \|u(t, \cdot)\|_{L^{q}(v)}^{q} \leq \log \|\varphi\|_{L^{q}(v)}^{q}, \quad 0 \leq t \leq T .
$$

Since $T>0$ is arbitrary, we obtain the desired result easily. The proof is complete.

Theorem 3.5. Assume that (1.2) holds and $\alpha, \beta>0$. Let $u$ be the unique classical solution to (1.1) obtained in Theorem 2.2. Then,

$$
\|u(t, \cdot)\|_{L^{1}(v)} \geq \exp \left\{e^{\beta t} \log \|\varphi\|_{L^{1}(v)}\right\}, \quad t \geq 0 .
$$

Proof. Let $u_{n}$ be the function defined by (3.12). Similarly to the arguments of the proof of Theorem 3.4, we get

$$
\begin{aligned}
\frac{d}{d t}\left\|u_{n}(t, \cdot)\right\|_{L^{1}(v)}= & {\left[\int_{\mathbb{R}^{N}} L u_{n}(t, \cdot) d \nu+\beta \int_{\mathbb{R}^{N}} u_{n}(t, \cdot) \log u_{n}(t, \cdot) d \nu\right] } \\
& -\int_{\mathbb{R}^{N}}\left(u(t, \cdot) L \rho_{n}+D u(t, \cdot) \cdot D \rho_{n}+\beta u_{n}(t, \cdot) \log \rho_{n}\right) d v \\
:= & \hat{I}(t)-\hat{J}(t) .
\end{aligned}
$$

By (3.2) and the Jensen inequality, we have

$$
\widehat{I}(t) \geq\left\|u_{n}(t, \cdot)\right\|_{L^{1}(v)} \log \left\|u_{n}(t, \cdot)\right\|_{L^{1}(v)}, \quad t>0 .
$$

Next, let us fix $T>0$ arbitrarily. By Lemma 3.3, it is easy to see that

$$
\hat{\theta}_{n}(T):=\sup \{|\hat{J}(T)| \mid t \in[0, T]\} \longrightarrow 0 \quad \text { as } n \longrightarrow \infty .
$$

Then the function $g_{n}(t)$ defined by

$$
g_{n}(t)=\left\|u_{n}(t, \cdot)\right\|_{L^{1}(v)}
$$

satisfies

$$
\frac{d}{d t} g_{n}(t) \geq \beta g_{n}(t) \log g_{n}(t)-\hat{\theta}_{n}(T), \quad 0<t<T
$$

Similarly to (3.19), we note that for each $T>0$ there exists a constant $\epsilon_{T}>0$ such that

$$
g_{n}(t) \geq \epsilon_{T}>0, \quad 0 \leq t \leq T \text {. }
$$

Then, by (3.28), we obtain

$$
\frac{d}{d t} \log g_{n}(t) \geq \beta \log g_{n}(t)-\frac{\hat{\theta}_{n}(T)}{\epsilon_{T}}, \quad 0<t<T .
$$


From this inequality, we have

$$
e^{-\beta t} \log \left\|u_{n}(t, \cdot)\right\|_{L^{1}(v)} \geq \log \left\|u_{n}(0, \cdot)\right\|_{L^{1}(v)}-\frac{\hat{\theta}_{n}(T)}{\beta \epsilon_{T}}\left(1-e^{-\beta t}\right), \quad 0 \leq t \leq T .
$$

Letting $n \rightarrow \infty$ and using the Lebesgue's dominated convergence theorem, we conclude that

$$
e^{-\beta t} \log \|u(t, \cdot)\|_{L^{1}(v)} \geq \log \|\varphi\|_{L^{1}(v)}, \quad 0 \leq t \leq T .
$$

Since $T>0$ is arbitrary, we obtain the desired result easily. The proof is complete.

\section{The main results}

In this section, we will state our main results of this paper and prove them. For $\alpha, \beta>0$, we write (1.1) $)_{\alpha, \beta}$ for the parabolic problem (1.1) to emphasize the dependence on $\alpha, \beta>0$. We denote by $u_{\varphi, \alpha, \beta}$ the unique solution of $(1.1)_{\alpha, \beta}$ for $\varphi$ with (1.2).

Definition 4.1. Let $\alpha, \beta>0$ and $q>1$. We define $\Gamma_{q}(\alpha, \beta)$ by

$$
\Gamma_{q}(\alpha, \beta)=\left\{\varphi \mid \varphi(\cdot)>0, \log \varphi \in \operatorname{Lip}\left(\mathbb{R}^{N}\right), \lim _{t \rightarrow \infty}\left\|u_{\varphi, \alpha, \beta}(t, \cdot)\right\|_{L^{q}(v)}=0\right\},
$$

where $v$ is the Gaussian measure of (3.1). We call $\Gamma_{q}(\alpha, \beta)$ the basin of $(1.1)_{\alpha, \beta}$.

We are interested in the problem to compare $\Gamma_{q}(\alpha, \beta)$ with the ball of the radius $\delta>0$ defined by

$$
B_{q}(\delta)=\left\{\varphi \mid \varphi(\cdot)>0, \log \varphi \in \operatorname{Lip}\left(\mathbb{R}^{N}\right),\|\varphi\|_{L^{q}(v)}<\delta\right\} .
$$

Theorem 4.2. Let $\alpha, \beta>0$ and $q>1$. Then,

$$
\Gamma_{q}(\alpha, \beta) \subset B_{1}(1)
$$

Proof. Let $\varphi \notin B_{1}(1)$. Then, $\|\varphi\|_{L^{1}(v)} \geq 1$. Since $v$ is the probability measure, it follows from Theorem 3.5 that

$$
\liminf _{t \rightarrow \infty}\left\|u_{\varphi, \alpha, \beta}(t, \cdot)\right\|_{L^{q}(v)} \geq \liminf _{t \rightarrow \infty}\left\|u_{\varphi, \alpha, \beta}(t, \cdot)\right\|_{L^{1}(v)} \geq 1 .
$$

This implies that $\varphi \notin \Gamma_{q}(\alpha, \beta)$. The proof is complete.

Now, we state the main result of this paper.

Theorem 4.3. Let $\beta>0$ and $q>1$. Then, we have the following.

(i) There exists a constant $\alpha_{0}=\alpha_{0}(\beta, q)\left(\beta / 2<\alpha_{0}\right)$ such that

$$
B_{q}(1) \subset \Gamma_{q}(\alpha, \beta), \quad \alpha \geq \alpha_{0} .
$$

(ii) For each $0<\delta \leq 1$, there exists a constant $\alpha_{1}=\alpha_{1}(\beta, \delta, q)\left(0<\alpha_{1}<\beta / 2\right)$ such that

$$
B_{q}(\delta) \not \subset \Gamma_{q}(\alpha, \beta), \quad 0<\alpha \leq \alpha_{1} .
$$


By Theorem 4.3, we see that the Ornstein-Uhlenbeck operator $L$ contributes to enlargement of the basin. Indeed, if $\alpha \geq \alpha_{0}$, then the basin is large enough to include $B_{q}(1)$. On the other hand, if $0<\alpha \leq \alpha_{1}$, the basin is small enough not to include $B_{q}(\delta)$.

Proof. (i) Let

$$
\alpha_{0}=\frac{q \beta}{2(q-1)}
$$

When $\alpha \geq \alpha_{0}$, we get $q \geq 2 \alpha /(2 \alpha-\beta)$. Hence (i) follows from Theorem 3.4.

(ii) Let

$$
\alpha_{1}=\frac{\beta \delta^{2 q / N} e^{-q}}{2} .
$$

We will construct $\varphi_{1} \in B_{q}(\delta)$ such that $\varphi_{1} \notin \Gamma_{q}(\alpha, \beta)$ for $0<\alpha \leq \alpha_{1}$. For $0<\alpha \leq \alpha_{1}$, we set

$$
\rho(x)=\exp \left\{-\left(\frac{\beta}{2}-\alpha\right)\left(|x|^{2}-\frac{N}{\beta}\right)\right\}, \quad x \in \mathbb{R}^{N} .
$$

It is easy to see that

$$
\|\rho\|_{L^{q}(v)}=\exp \left\{\frac{N(\beta-2 \alpha)}{2 \beta}\right\}\left[\frac{2 \alpha}{q(\beta-2 \alpha)+2 \alpha}\right]^{N / 2 q} .
$$

Since $0<\alpha \leq \alpha_{1}$, we get $q(\beta-2 \alpha)+2 \alpha \geq \beta$. Hence, for $0<\alpha \leq \alpha_{1}$, we see that

$$
\|\rho\|_{L^{q}(v)} \leq \exp \left\{\frac{N(\beta-2 \alpha)}{2 \beta}\right\}\left(\frac{2 \alpha}{\beta}\right)^{N / 2 q} \leq \exp \left\{\frac{N}{2}-\frac{\alpha N}{\beta}\right\} \delta e^{-N / 2}<\delta .
$$

Now, choose $C>0$ so that $e^{C}\|\rho\|_{L^{q}(v)}<\delta$. This is possible by (4.11). We define the function $u_{0}$ by

$$
u_{0}(t, x)=\rho(x) \exp \left\{C e^{\beta t}\right\}, \quad(t, x) \in[0, \infty) \times \mathbb{R}^{N}
$$

We set

$$
\varphi_{0}(x):=u_{0}(0, x)=\rho(x) e^{C} .
$$

Then, it is easy to see that $\mathcal{u}_{0}$ is a solution of $(1.1)_{\alpha, \beta}$ with $\varphi=\varphi_{0}$. Furthermore, we have

$$
\left\|\varphi_{0}\right\|_{L^{q}(v)}<\delta, \quad \lim _{t \rightarrow \infty} u_{0}(t, x)=+\infty\left(x \in \mathbb{R}^{N}\right) .
$$

However, note that $\varphi_{0}$ does not fulfill (1.2). Hence, we need the following device.

First of all, let us choose $R>0$ so that

$$
R>\sqrt{\frac{2 C}{\beta-2 \alpha}+\frac{N}{\beta}}, \quad v(|x|>R)<\delta^{q}-\left\|\varphi_{0}\right\|_{L^{q}(v)}^{q} .
$$


This is possible by (4.14), because $\nu(|x|>\widehat{R}) \rightarrow 0(\hat{R} \rightarrow \infty)$. We set

$$
\psi_{0}(x):=\log \varphi_{0}(x)=C-\left(\frac{\beta}{2}-\alpha\right)\left(|x|^{2}-\frac{N}{\beta}\right) .
$$

Then, it is easy to see that

$$
\psi_{0}(x)<0, \quad|x|>R
$$

Next, we choose $\chi \in C^{\infty}\left(\mathbb{R}^{N}\right)$ such that $0 \leq \chi(\cdot) \leq 1$ on $\mathbb{R}^{N}$ and

$$
\chi(x)= \begin{cases}1, & |x| \leq R \\ 0, & |x| \geq R+1\end{cases}
$$

Then, we define the functions $\psi_{1}$ and $\varphi_{1}$,

$$
\psi_{1}(x)=\chi(x) \psi_{0}(x), \quad \varphi_{1}(x)=\exp \left\{\psi_{1}(x)\right\}, \quad x \in \mathbb{R}^{N} .
$$

It is clear to see that $\varphi_{1}$ fulfills (1.2). By (4.17), we have

$$
\psi_{1}(x) \geq \psi_{0}(x), \quad x \in \mathbb{R}^{N}
$$

Let $u_{1}=u_{\alpha, \beta, \varphi_{1}}$ which is ensured by Theorem 2.2. By (4.15)-(4.17), we see that

$$
\left\|\varphi_{1}\right\|_{L^{q}(v)}^{q}=\int_{|x| \leq R} \exp \left\{q \psi_{0}\right\} d \nu+\int_{|x|>R} \exp \left\{q \chi \psi_{0}\right\} d \nu \leq\left\|\varphi_{0}\right\|_{L^{q}(v)}^{q}+\nu(|x|>R)<\delta^{q} .
$$

Hence, we see that $\varphi_{1} \in B_{q}(\delta)$.

On the other hand, for $j=1,2$, define $\eta_{j}(j=0,1)$ by $\eta_{j}(t, x)=e^{-\beta t} \log u_{j}(t, x)$. Since $\eta_{j}$ satisfies

$$
\begin{gathered}
\left(\eta_{j}\right)_{t}=\frac{1}{2} \Delta \eta_{j}-\alpha x \cdot D \eta_{j}+\frac{1}{2} e^{\beta t}\left|D \eta_{j}\right|^{2} \text { in }(0, \infty) \times \mathbb{R}^{N} \\
\eta_{j}(0, \cdot)=\psi_{j}(\cdot) \quad \text { in } \mathbb{R}^{N}
\end{gathered}
$$

we obtain on $(0, \infty) \times \mathbb{R}^{N}$,

$$
\left(\eta_{1}-\eta_{0}\right)_{t}=\frac{1}{2} \Delta\left(\eta_{1}-\eta_{0}\right)+\left[-\alpha x+\frac{1}{2} e^{\beta t}\left(D \eta_{1}+D \eta_{0}\right)\right] \cdot D\left(\eta_{1}-\eta_{0}\right) .
$$

By Theorem 2.2, we see that for any $T>0$ there exists a constant $K_{T}$ such that

$$
\begin{gathered}
\left|-\alpha x+\frac{1}{2} e^{\beta t}\left(D \eta_{1}+D \eta_{0}\right)(t, x)\right| \leq K_{T}(1+|x|), \quad(t, x) \in(0, T] \times \mathbb{R}^{N}, \\
\eta_{1}(t, x)-\eta_{0}(t, x) \geq-K_{T}(1+|x|), \quad(t, x) \in[0, T] \times \mathbb{R}^{N} .
\end{gathered}
$$


By (4.20) and the comparison theorem for parabolic equations (cf. [5, Theorem 9, page 43]) we deduce that

$$
\eta_{1}(t, x)-\eta_{0}(t, x) \geq 0, \quad(t, x) \in[0, \infty) \times \mathbb{R}^{N} .
$$

Hence, by (4.14), we see that

$$
\lim _{t \rightarrow \infty} u_{1}(t, x)=+\infty, \quad x \in \mathbb{R}^{N}
$$

By Fatou's lemma, we have

$$
\lim _{t \rightarrow \infty}\left\|u_{1}(t, \cdot)\right\|_{L^{q}(v)}=+\infty
$$

The proof is complete.

\section{References}

[1] S. Cerrai, Second Order PDE's in Finite and Infinite Dimension. A Probabilistic Approach, Lecture Notes in Mathematics, vol. 1762, Springer, Berlin, 2001.

[2] G. Da Prato and B. Goldys, Elliptic operators on $\mathbb{R}^{d}$ with unbounded coefficients, Journal of Differential Equations 172 (2001), no. 2, 333-358.

[3] _ Erratum: Elliptic operators on $\mathbb{R}^{d}$ with unbounded coefficients, Journal of Differential Equations 184 (2002), no. 2, 620.

[4] G. Da Prato and A. Lunardi, On the Ornstein-Uhlenbeck operator in spaces of continuous functions, Journal of Functional Analysis 131 (1995), no. 1, 94-114.

[5] A. Friedman, Partial Differential Equations of Parabolic Type, Prentice-Hall, New Jersey, 1964.

[6] Y. Fujita, H. Ishii, and P. Loreti, Asymptotic solutions of viscous Hamilton-Jacobi equations with Ornstein-Uhlenbeck operator, to appear in Communications in PDE.

[7] L. Gross, Logarithmic Sobolev inequalities, American Journal of Mathematics 97 (1975), no. 4, 1061-1083.

[8] A. A. Samarskii, V. A. Galaktionov, S. P. Kurdyumov, and A. P. Mikhailov, Blow-Up in Quasilinear Parabolic Equations, De Gruyter Expositions in Mathematics, vol. 19, Walter de Gruyter, Berlin, 1995.

Yasuhiro Fujita: Department of Mathematics, Toyama University, Toyama 930-8555, Japan E-mail address: yfujita@sci.toyama-u.ac.jp 